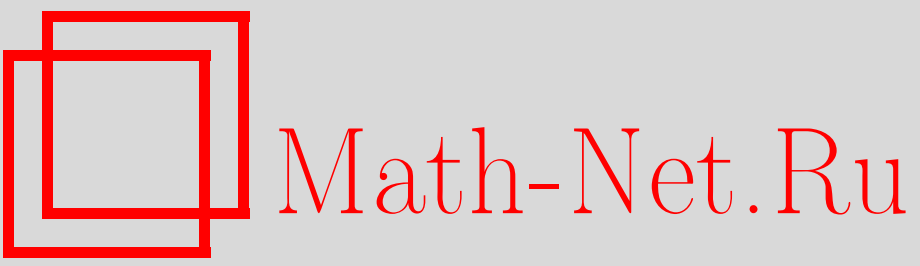

М. Маньяс, Л. Мартинес Алонсо, Е. Медина, КП-теория сетей Егорова, ТМФ, 2001, том 127, номер 3, 457-464

DOI: https://doi.org/10.4213/tmf473

Использование Общероссийского математического портала Math-Net.Ru подразумевает, что вы прочитали и согласны с пользовательским соглашением

http://www.mathnet.ru/rus/agreement

Параметры загрузки:

IP : 54.198 .187 .58

26 апреля 2023 г., 15:54:01 
ТЕОРЕТИЧЕСКАЯ

И МАТЕМАТИЧЕСКАЯ

ФИЗИКА

Том 127, № 3

июнь, 2001

(C) 2001 г. $\quad$ М. Маньяс*, Л. Мартинес Алонсо*, Е. Медина ${ }^{\dagger}$

\section{КП-ТЕОРИЯ СЕТЕЙ ЕГОРОВА}

Теория многокомпонентной КП-иерархии используется для характеризации явных примеров сетей Егорова. Особенно эффективным оказывается метод $\bar{\partial}$-одевания для пропагаторов Коши.

\section{1. ВВЕДЕНИЕ}

Два десятилетия тому назад было обнаружено, что теория ортогональных сетей

$$
d s^{2}=\sum_{i=1}^{M} H_{i}^{2}\left(d u_{i}\right)^{2}
$$

тесно связана с теорией интегрируемых систем гидродинамического типа в размерности $1+1$ [1]-[3]. Более того, частный тип ортогональных сетей (Ә-инвариантные сети Егорова) оказался актуальным при классификации топологических квантовых теорий поля [4]. В настоящей работе описываются некоторые недавние достижения в применении теории Кадомцева-Петвиашвили (КП) к теории сетей Егорова.

Рассмотрим систему дифференциальных уравнений в частных производных [5]-[7]

$$
\begin{aligned}
& \frac{\partial \beta_{i j}}{\partial u_{k}}-\beta_{i k} \beta_{k j}=0, \quad i, j, k=1, \ldots, N, \text { если } i, j, k \text { различны, } \\
& \frac{\partial \beta_{i j}}{\partial u_{i}}+\frac{\partial \beta_{j i}}{\partial u_{j}}+\sum_{\substack{k=1, \ldots, N \\
k \neq i, j}} \beta_{k i} \beta_{k j}=0, \quad i, j=1, \ldots, N, \quad i \neq j,
\end{aligned}
$$

где $\beta_{i j}:=H_{i}^{-1} \partial H_{j} / \partial u_{i}$, при условиях

$$
\beta_{i j}=\beta_{j i} ; \quad \partial H_{i}=0, \quad \partial:=\sum_{j} \frac{\partial}{\partial u_{j}} .
$$

* Departamento de Física Teórica II, Universidad Complutense, Madrid, Spain. E-mail: manuel@darboux.fis.ucm.es; luism@eucmos.sim.ucm.es

${ }^{\dagger}$ Departamento de Matemáticas, Universidad de Cádiz, Cádiz, Spain. E-mail: elena.medina@uca.es 
Важная в данном контексте математическая структура - класс многообразий Фробениуса - была предложена в работах [2], [4], [8]-[10]. Локально многообразие Фробениуса определяется [4] плоской метрикой

$$
d s^{2}=\sum_{i, j=1}^{N} \eta^{i j} d x_{i} d x_{j}
$$

и структурой коммутативной ассоциативной алгебры

$$
\partial_{i} \cdot \partial_{j}=\sum_{k} c_{i j}^{k}(x) \partial_{k}, \quad \partial_{i}:=\frac{\partial}{\partial x^{i}}, \quad x^{i}:=\sum_{k} \eta^{i k} x_{k},
$$

с единицей $\partial_{1}$. Метрика $d s^{2}$ должна быть инвариантной по отношению к данному произведению, а деформированная связность

$$
\nabla_{i} X^{j}:=\partial_{i} X^{j}+z \sum_{k} c_{i k}^{j}(\mathbf{x}) X^{k}
$$

где $z$ - спектральный параметр, должна иметь нулевую кривизну.

Значительная часть структуры многообразия Фробениуса содержится в системах деформированных плоских координат $\theta_{k}(z, \mathbf{x})$ [4], [8]-[10] для связности (1)

$$
\nabla_{i} \nabla_{j} \theta_{k}=0
$$

или, эквивалентным образом,

$$
\partial_{i} \partial_{j} \theta_{k}=z \sum_{l} c_{i j}^{l}(\mathbf{x}) \partial_{l} \theta_{k}
$$

С другой стороны, из сделанных допушений относительно $\left(\eta, c_{i j}^{k}(\mathbf{x})\right)$ следует, что существует такая функция $F=F(\mathbf{x})$ (функиия свободной энергии), что

$$
c_{i j k}=\partial_{i} \partial_{j} \partial_{k} F
$$

и в силу свойства ассоциативности алгебры функция $F$ удовлетворяет уравнениям Виттена-Дайкграфа-Э. Верлинде-Х. Верлинде (ВДВВ) [11], [12]

$$
\sum_{r, s} \partial_{i} \partial_{j} \partial_{r} F \eta^{r s} \partial_{s} \partial_{m} \partial_{k} F=\sum_{r, s} \partial_{i} \partial_{m} \partial_{s} F \eta^{s r} \partial_{r} \partial_{j} \partial_{k} F
$$

В системе деформированных плоских координат, нормированных условием

$$
\theta_{i}(0, \mathbf{x})=x_{i}, \quad i=1, \ldots, N
$$

из уравнения (2) вытекает [4], что функцию свободной энергии можно получить из уравнения

Более того, коэффициенты разложения

$$
\partial_{i} F(\mathbf{x})=\frac{\partial \theta_{i}}{\partial z}(0, \mathbf{x})
$$

$$
\theta_{i}(z, \mathbf{x})=\sum_{p \geqslant 0} h_{i, p}(\mathbf{x}) z^{p}
$$

определяют бесконечное семейство функционалов

$$
H_{i, p}[\mathbf{x}]:=\int h_{i, p+1}(\mathbf{x}) d t,
$$

которые находятся в инволюции по отношению к скобке Пуассона

$$
\left\{x^{i}\left(t_{1}\right), x^{j}\left(t_{2}\right)\right\}:=\eta^{i j} \delta^{\prime}\left(t_{1}-t_{2}\right) .
$$

Соответствуюшие гамильтоновы системы составляют интегрируемую иерархию систем гидродинамического типа. 


\section{2. КП-ИЕРАРХИИ И ГРАССМАННИАН}

$N$-компонентную КП-иерархию можно ввести как семейство потоков в бесконечномерном грассманниане [13], [14]. Пусть $D(r)$ и $\gamma(r)$ обозначают соответственно диск $\{z \in \mathbb{C}:|z| \leqslant r\}$ и его границу $\{z \in \mathbb{C}:|z|=r\}$ и пусть $H_{\gamma(r)}$-множество рядов Лорана

$$
\sum_{n=-\infty}^{\infty} a_{n} z^{n}
$$

с коэффициентами $a_{n} \in M_{N}(\mathbb{C})$ (кольцо комплексных $(N \times N)$-матриц), которые сходятся на окружности $\gamma(r)$. В дальнейшем нам понадобятся два различных грассманниана $\mathrm{Gr}_{\gamma(r)}$ и $\mathrm{Gr}_{\gamma(r)}^{*}$.

ОПРЕДЕЛЕНИЕ 1. Элементы из $\mathrm{Gr}_{\gamma(r)}$ являются такими подмножествами $W$ в $H_{\gamma(r)}$, что

1) $W$ есть левый $M_{N}(\mathbb{C})$-модуль,

2) проекция оператора $P_{+} W \rightarrow H_{\gamma(r)}^{+}$из $W$ в

$$
H_{\gamma(r)}^{+}=\left\{w \in H_{\gamma(r)}: w=\sum_{n=0}^{\infty} a_{n} z^{n}\right\}
$$

является биекцией.

Аналогично грассманниан $\mathrm{Gr}_{\gamma(r)}^{*}$ состоит из таких подмножеств $V$ в $H_{\gamma(r)}$, что

$\left.1^{*}\right) V$ есть правый $M_{N}(\mathbb{C})$-модуль,

$\left.2^{*}\right)$ проекция оператора $P_{+}: V \rightarrow H_{\gamma(r)}^{+}$является биекцией.

Имеется отображение

$$
\mathrm{Gr}_{\gamma(r)} \stackrel{*}{\rightarrow} \mathrm{Gr}_{\gamma(r)}^{*}, \quad W \mapsto W^{*}
$$

такое, что для всякого заданного $W \in \mathrm{Gr}_{\gamma(r)}$ подпространство $W^{*} \in \mathrm{Gr}_{\gamma(r)}^{*}$ является множеством тех элементов $v \in H_{\gamma(r)}$, которые удовлетворяют уравнению

$$
\int_{\gamma(r)} w(z) v(z) d z=0 \quad \forall w \in W
$$

Типичные элементы грассманниана возникают в рамках $\bar{\partial}$-метода. Для заданной подходящей $(N \times N)$-матричной обобшенной функции $R\left(z, z^{\prime}\right)$ с носителем в $D(r) \times D(r)$ соответствуюший элемент $W \in \mathrm{Gr}_{\gamma(r)}$ дается множеством ограничений на $\gamma(r)$ решений $w(z)$ уравнения

$$
\frac{\partial w}{\partial \bar{z}}(z)=\int_{D(r)} w\left(z^{\prime}\right) R\left(z^{\prime}, z\right) d^{2} z^{\prime} .
$$

Тогда $W^{*} \in \mathrm{Gr}_{\gamma(r)}^{*}$ есть решение уравнения

$$
\frac{\partial v}{\partial \bar{z}}(z)=-\int_{D(r)} R\left(z, z^{\prime}\right) v\left(z^{\prime}\right) d^{2} z^{\prime} .
$$


ОПРЕДЕлЕниЕ 2. Связанная с заданным элементом $W \in \mathrm{Gr}_{\gamma}(r)$ KП-функиия Бейкера является единственным элементом $\psi \in W$, который допускает сходящееся разложение вида

$$
\psi(z, \mathbf{u})=\chi(z, \mathbf{u}) \psi_{0}(z, \mathbf{u}), \quad \chi(z, \mathbf{u})=I_{N}+\sum_{n \geqslant 1} \frac{a_{n}(\mathbf{u})}{z^{n}}, \quad \mathbf{u} \in \mathcal{U}(r)^{N}, \quad z \in \gamma(r) .
$$

Сопряэенная КП-функиия Бейкера есть единственный элемент $\psi^{*} \in W^{*}$ с разложением

$$
\psi^{*}(z, \mathbf{u})=\psi_{0}(z, \mathbf{u})^{-1} \chi^{*}(z, \mathbf{u}), \quad \chi^{*}(z, \mathbf{u})=I_{N}+\sum_{n \geqslant 1} \frac{a_{n}^{*}(\mathbf{u})}{z^{n}}, \quad \mathbf{u} \in \mathcal{U}(r)^{N}, \quad z \in \gamma(r)
$$

Здесь $I_{N}:=\sum_{i=1}^{N} E_{i}$ обозначает единичную матрицу в $M_{N}(\mathbb{C})$. Отметим, что при любых $\mathbf{u} \in \mathcal{U}(r)^{N}$ как $\chi(z, \mathbf{u})$, так и $\chi^{*}(z, \mathbf{u})$ являются аналитическими функциями $z$ в области $\mathbb{C} \backslash D(r)$.

Функция Бейкера удовлетворяет так называемой $N$-компонентной КП-иерархии. Эта иерархия является бесконечной системой линейных уравнений

$$
\frac{\partial \psi}{\partial u_{i, n}}=P_{i, n}(\mathbf{u}, \partial) \psi, \quad i=1, \ldots, N, \quad n \geqslant 1, \quad \partial:=\partial_{1}+\cdots+\partial_{N},
$$

где $P_{i, n}(\mathbf{u}, \partial)$ - семейство линейных по $\partial$ дифференциальных операторов.

Первые несколько членов иерархии (5) имеют вид

$$
\frac{\partial \psi}{\partial u_{i, 1}}=E_{i} \partial \psi+\left[a_{1}, E_{i}\right] \psi, \quad i=1, \ldots, N
$$

что можно переписать как

$$
\frac{\partial \boldsymbol{\psi}_{i}}{\partial u_{k}}=\beta_{i k} \boldsymbol{\psi}_{k}, \quad i \neq k
$$

где

$$
\psi_{i}:=\left(\psi_{i 1}, \ldots, \psi_{i N}\right), \quad u_{k}:=u_{k, 1}, \quad \beta=a_{1} .
$$

Сопряженная подобным образом функция Бейкера удовлетворяет линейной системе

$$
\frac{\partial \boldsymbol{\psi}_{j}^{*}}{\partial u_{k}}=\boldsymbol{\psi}_{k}^{*} \beta_{k j}, \quad j \neq k
$$

где

$$
\boldsymbol{\psi}_{i}^{*}:=\left(\begin{array}{c}
\psi_{1 i}^{*} \\
\vdots \\
\psi_{N i}^{*}
\end{array}\right) .
$$

Условия совместности как уравнений (6), так и уравнений (7) приводят к системе уравнений Дарбу для сопряженной сети. Более того, из уравнений (6) и (7) видно, что с набором коэффициентов врашения $\beta_{i j}$ связано семейство сопряженных сетей с касательными векторами и коэффициентами Ламе вида $\left(\mathbf{X}_{i}\right)_{j}:=\mathbf{X}_{i j}$ и $H_{i}=\mathbf{H}_{l i}, l=1, \ldots, N$, где

$$
\mathbf{X}(\mathbf{u}):=\int_{\mathbb{C}} \psi(z, \mathbf{u}) N(z) d^{2} z, \quad \mathbf{H}(\mathbf{u}):=\int_{\mathbb{C}} M(z) \psi^{*}(z, \mathbf{u}) d^{2} z .
$$

Здесь $N(z)$ и $M(z)$ - подходяшие $(N \times N)$-матричные обобшенные функции. 


\section{3. ПРОПАГАТОР КОШИ}

ОПРЕДЕлЕНИЕ 3. Связанный с заданным элементом $W \in \mathrm{Gr}_{\gamma(r)}$ nponazamop Kouи есть функция Грина $\Psi=\Psi\left(z, z^{\prime}, \mathbf{u}\right) \bar{\partial}$-оператора

$$
\frac{\partial \Psi}{\partial \bar{z}}\left(z, z^{\prime}, \mathbf{u}\right)=\pi \delta\left(z-z^{\prime}\right), \quad z, z^{\prime} \in \mathbb{C} \backslash D(r), \quad \mathbf{u} \in \mathcal{U}(\infty)^{N}
$$

удовлетворяющая следующим граничным условиям:

1) для каждого фиксированного $\mathbf{u} \in \mathcal{U}(\infty)^{N}$ и $z^{\prime} \in \mathbb{C} \backslash D(r)$ ограничение $\Psi$ на $\gamma(r)$, рассматриваемое как функция $z$, является элементом $W$;

2) при $z \rightarrow \infty$ имеем

$$
\Psi\left(z, z^{\prime}, \mathbf{u}\right)=\mathcal{O}\left(\frac{1}{z}\right) \psi_{0}(z, \mathbf{u})
$$

Следуюшая теорема [2] связывает пропагатор Коши с функцией Бейкера. Используем обозначения

$$
[z]=\left([z]_{1}, \ldots,[z]_{N}\right), \quad[z]_{i}=\left(\frac{1}{z}, \ldots, \frac{1}{n z^{n}}, \ldots\right) .
$$

Теорема 1. Пропагатор Коши, связанный с әлементом $W$ из $\mathrm{Gr}_{\gamma(r)}$, можнно записать в терминах волновых функиий $К П \psi и \psi^{*}$ как

$$
\Psi\left(z, z^{\prime}, \mathbf{u}\right)=\left\{\begin{array}{lll}
-\frac{1}{z^{\prime}} \psi^{*}\left(z^{\prime}, \mathbf{u}\right) \psi\left(z, \mathbf{u}+\left[z^{\prime}\right]\right) & \text { npu } & |z| \leqslant\left|z^{\prime}\right| \\
\frac{1}{z} \psi^{*}\left(z^{\prime}, \mathbf{u}-[z]\right) \psi(z, \mathbf{u}) & n p u & \left|z^{\prime}\right| \leqslant|z| .
\end{array}\right.
$$

Матричные әлементы $\Psi$ удовлетворяют дифференциальным уравнениям

$$
\frac{\partial \Psi_{j k}}{\partial u_{i}}\left(z, z^{\prime}, \mathbf{u}\right)=\psi_{j i}^{*}\left(z^{\prime}, \mathbf{u}\right) \psi_{i k}(z, \mathbf{u})
$$

Из уравнения (8) следует, что сетевая функция сопряженной сети с касательными векторами и коэффициентами Ламе, задаваемыми соответственно формулами $\left(\mathbf{X}_{i}\right)_{j}:=$ $\mathbf{X}_{i j}$ и $H_{i}=\mathbf{H}_{l i}, l=1, \ldots, N$, выражается как $l$-я строка в матричной функции

$$
\mathbf{x}:=\int_{\mathbb{C} \times \mathbb{C}} M\left(z^{\prime}\right) \Psi\left(z, z^{\prime}\right) N(z) d^{2} z d^{2} z^{\prime}+\mathbf{x}_{0},
$$

где $\mathbf{x}_{0}-$ произвольная постоянная матрица.

\section{4. РЕДУКЦИЯ ЕГОРОВА}

ОПРЕДЕЛЕНИЕ 4. Элемент $W \in \mathrm{Gr}_{\gamma(r)}$ удовлетворяет редукиии Егорова, если

1) для каждого $w \in W$ функция $\widetilde{w}(z):=z w(z)$ также лежит в $W$;

2) для каждого $v \in W^{*}$ функция $\tilde{v}(z):=v(-z)^{\mathrm{t}}$ лежит в $W$.

Следуюшая теорема доказана в работе [15]. 
ТеОрема 2. Если әлемент $W \in \mathrm{Gr}_{\gamma}(r)$ удовлетворяет редукиии Егорова, то для любой несингулярной матрищъ $\mathcal{N}$ функиии

$$
\theta_{i}(z, \mathfrak{u}):=\left(\mathcal{N}^{\mathrm{t}}\left(\Psi(z, 0, \mathfrak{u})-\frac{1}{z}\right) \mathcal{N}\right)_{1 i}, \quad i=1, \ldots, N
$$

являются системой нормированных деформированных плоских координат для многообразия Фробениуса, определенного:

1) д-инвариантной метрикой Егорова

$$
d s^{2}=\sum_{i=1}^{N} H_{i}^{2}\left(d u_{i}\right)^{2}, \quad H_{i}(\mathfrak{u}):=(\psi(0, \mathfrak{u}) \mathcal{N})_{i 1} ;
$$

2) системой плоских координат

$$
\begin{aligned}
x_{i} & :=\theta_{i}(0, \mathfrak{u}), \quad i=1, \ldots, N \\
d s^{2} & =\sum_{i, j=1}^{N} \eta^{i j} d x_{i} d x_{j}, \quad \eta=\left(\mathcal{N}^{\mathrm{t}} \mathcal{N}\right)^{-1}
\end{aligned}
$$

3) структурныцми константами

$$
c_{i j}^{l}=\sum_{k=1}^{N} \frac{\partial u_{k}}{\partial x^{i}} \frac{\partial u_{k}}{\partial x^{j}} \frac{\partial x^{l}}{\partial u_{k}} .
$$

Отметим, что из формул (9) и (4) следует, что каждњй элемент $W \in \mathrm{Gr}_{\gamma(r)}$, удовлетворяющий редукции Егорова, определяет иерархию систем гидродинамического типа с гамильтоновыми плотностями

$$
h_{i, p}(\mathbf{x})=\left.\frac{1}{(p+1) !} \frac{\partial^{p+1}}{\partial z^{p+1}}\left(\mathcal{N}^{\mathrm{t}} z \Psi(z, 0, \mathfrak{u}) \mathcal{N}\right)_{1 i}\right|_{z=0} .
$$

\section{5. ОДЕВАНИЕ СОПРЯЖЕННЫХ СЕТЕЙ}

Рассмотрим теперь метод одевания для сопряженных сетей [16]. Пусть $D(r)$ и $D(\tilde{r})-$ два диска с центрами в начале координат, $r<\tilde{r}$. Пусть $\gamma(r)$ и $\gamma(\tilde{r})-$ границы этих дисков, а $A$ - кольцо $D(\tilde{r})-D(r)$.

ОПРЕДЕЛЕниЕ 5. Матричная обобшенная функция $R=R\left(z, z^{\prime}\right)$ с носителем в $A \times A$ определяет одевающее преобразование

$$
T_{R}: \operatorname{Gr}_{\gamma(r)} \mapsto \operatorname{Gr}_{\gamma(\tilde{r})}, \quad W \mapsto \widetilde{W},
$$

где для каждого $W \in \operatorname{Gr}_{\gamma(r)}$ соответствующий элемент $\widetilde{W} \in \operatorname{Gr}_{\gamma(\tilde{r})}$ дается граничными значениями на $\gamma(\tilde{r})$ матричных функций $w=w(z)$, удовлетворяющих $\bar{\partial}$-уравнениям

$$
\frac{\partial w}{\partial \bar{z}}(z)=\int_{A} w\left(z^{\prime}\right) R\left(z^{\prime}, z\right) d^{2} z^{\prime}, \quad z \in A,
$$

причем ограничение $w$ на $\gamma(r)$ является элементом из $W$. 
Для случая сепарабельного ядра

$$
R\left(z, z^{\prime}\right)=\pi \sum_{k=1}^{m} \sum_{\ell=1}^{n} C_{k \ell} f_{k}(z) g_{\ell}\left(z^{\prime}\right)
$$

одевание пропагатора Коши можно выполнить явно. В этом случае $C_{k \ell}$ - постоянные комплексные $(N \times N)$-матрицы, а $f_{k}$ и $g_{\ell}$ - скалярные распределения. Чтобы определить соответствуюшее преобразование, полезно ввести обозначения

$$
\begin{aligned}
\mu_{k}(z) & :=\int_{A} \Psi\left(z^{\prime}, z\right) f_{k}\left(z^{\prime}\right) d^{2} z^{\prime}, \quad k=1, \ldots, m, \\
\nu_{\ell}(z) & :=\int_{A} \Psi\left(z, z^{\prime}\right) g_{\ell}\left(z^{\prime}\right) d^{2} z^{\prime}, \quad \ell=1, \ldots, n, \\
\omega_{\ell k} & :=\int_{A \times A} \Psi\left(z^{\prime}, z^{\prime \prime}\right) f_{k}\left(z^{\prime}\right) g_{\ell}\left(z^{\prime \prime}\right) d^{2} z^{\prime} d^{2} z^{\prime \prime}, \quad k=1, \ldots, m, \quad \ell=1, \ldots, n .
\end{aligned}
$$

Определим также матрицы

$$
\begin{gathered}
\boldsymbol{\mu}=\left(\mu_{1}, \ldots, \mu_{m}\right): A \rightarrow M_{N \times m N}(\mathbb{C}), \quad \boldsymbol{\nu}=\left(\begin{array}{c}
\nu_{1} \\
\vdots \\
\nu_{n}
\end{array}\right): A \rightarrow M_{n N \times N}(\mathbb{C}), \\
\mathbf{C}=\left(C_{k \ell}\right) \in M_{m N \times n N}(\mathbb{C}), \quad \boldsymbol{\omega}=\left(\omega_{\ell k}\right) \in M_{n N \times m N}(\mathbb{C}) .
\end{gathered}
$$

Тогда имеем [16]

$$
\widetilde{\Psi}\left(z, z^{\prime}\right)=\Psi\left(z, z^{\prime}\right)+\boldsymbol{\mu}\left(z^{\prime}\right) \mathbf{C}(1-\boldsymbol{\omega} \mathbf{C})^{-1} \boldsymbol{\nu}(z) .
$$

\section{6. ОДЕВАНИЕ СЕТЕЙ ЕГОРОВА}

Редукция Егорова сохраняется при одевании, если

$$
v \in \widetilde{W}^{*} \Rightarrow v^{\mathrm{t}}(-z) \in \widetilde{W} .
$$

Для ядра, удовлетворяюшего уравнениям

$$
\begin{aligned}
z R\left(z, z^{\prime}\right) & =z^{\prime} R\left(-z^{\prime},-z\right)^{\mathrm{t}}, \\
R\left(z, z^{\prime}\right) & =R\left(-z^{\prime},-z\right)^{\mathrm{t}},
\end{aligned}
$$

соответствуюшее преобразование одевания сохраняет эту редукцию. Более того, из этих условий следует, что

$$
R\left(z, z^{\prime}\right)=R_{0}(z) \delta\left(z-z^{\prime}\right), \quad R_{0}(z)=R_{0}(-z)^{\mathrm{t}} .
$$

Сепарабельные ядра этого типа имеют вид

$$
R_{0}(z)=\pi \sum_{k=1}^{n}\left[C_{k} \delta\left(z-p_{k}\right)+C_{k}^{\mathrm{t}} \delta\left(z+p_{k}\right)\right]
$$


где $C_{k}$ - комплексные $(N \times N)$-матрицы и $p_{k} \in \mathbb{C}$, т.е.

$$
R\left(z, z^{\prime}\right)=\pi \sum_{k=1}^{n}\left[C_{k} \delta\left(z-p_{k}\right) \delta\left(z^{\prime}-p_{k}\right)+C_{k}^{\mathrm{t}} \delta\left(z+p_{k}\right) \delta\left(z^{\prime}+p_{k}\right)\right]
$$

Соответствующее одевающее преобразование, в котором могут возникнуть проблемы, связанные с сингулярностями, оказывается корректно определенным, если

$$
C_{k}^{2}=0, \quad k=1, \ldots, n .
$$

Явные примеры сетей Егорова и соответствуюших им многообразий Фробениуса можно, таким образом, охарактеризовать как вакуумные решения [15]. Например, функция свободной энергии

$$
\begin{aligned}
F\left(x_{1}, \ldots, x_{N}\right)= & \frac{1}{6} x_{1}^{3}+\frac{1}{p}\left(x_{2}^{2}+\cdots+x_{N}^{2}\right)+\frac{x_{1}}{2}\left(x_{2}^{2}+\cdots+x_{N}^{2}\right)+ \\
& +\frac{1}{6 p^{3}}\left[1+2 p^{2}\left(x_{2}^{2}+\cdots+x_{N}^{2}\right)\right] \sqrt{1-p^{2}\left(x_{2}^{2}+\cdots+x_{N}^{2}\right)}- \\
& -\sum_{j=2}^{N} \frac{x_{j}^{2}}{2 p} \ln \left[-\frac{2 x_{j}}{c_{j}} \frac{1+\sqrt{1-p^{2}\left(x_{2}^{2}+\cdots+x_{N}^{2}\right)}}{x_{2}^{2}+\cdots+x_{N}^{2}}\right]
\end{aligned}
$$

удовлетворяет уравнениям ассоциативности ВДВВ (3).

Благодарности. Работа выполнена при частичной финансовой поддержке Dirección General de Enseñanza Superior e Investigación Científica (проект PB98-0821).

\section{Список литературы}

[1] Б. А. Дубровин, С. П. Новиков. ДАН СССР. 1983. Т. 270. С. 781.

[2] Б. А. Дубровин, С. П. Новиков. УМН. 1989. Т. 44. № 6. С. 29.

[3] С. П. Царев. ДАН СССР. 1985. Т. 282. С. 534.

[4] B. Dubrovin. Nucl. Phys. B. 1992. V. 379. P. 627.

[5] G. Darboux. Leçons sur les systèmes orthogonaux et les coordenées curvilignes. Paris: Gauthier-Villars, 1910; Reprinted: Sceaux: Éditions Jacques Gabay, 1993.

[6] G. Lamé. Leçons sur la théorie des coordenées curvilignes et leurs diverses applications. Paris: Mallet-Bachalier, 1859.

[7] V.E. Zakharov. Duke Math. J. 1998. V. 94. Р. 103; B. Е. Захаров, С. В. Манаков. ДАН. 1998. T. 360. № 3. C. 324.

[8] B. Dubrovin. Geometry of 2D topological field theory. Preprint SISSA-89/94/FM, SISSA, Trieste, 1994; hep-th/9407018.

[9] B. Dubrovin, Y. Zhang. Commun. Math. Phys. 1998. V. 198. P. 311

[10] B. Dubrovin, Y. Zhang. Frobenius manifolds and Virasoro constraints. math.AG/9808048.

[11] E. Witten. Nucl. Phys. B. 1990. V. 340. P. 281.

[12] R. Dijkgraaf, E. Verlinde, H. Verlinde. Nucl. Phys. B. 1991. V. 325. P. 59.

[13] G. Segal, G. Wilson. Publ. Math. IHES. 1985. V. 61. P. 5.

[14] E. Witten. Commun. Math. Phys. 1988. V. 113. P. 529.

[15] M. Mañas, L. Martínez Alonso, E. Medina. J. Phys. A. 2000. V. 33. P. 7181.

[16] M. Mañas, L. Martínez Alonso, E. Medina. J. Phys. A. 2000. V. 33. P. 2871. 